This article was downloaded by: [National Taiwan University]

On: 30 March 2009

Access details: Access Details: [subscription number 769150938]

Publisher Taylor \& Francis

Informa Ltd Registered in England and Wales Registered Number: 1072954 Registered office: Mortimer House, 37-41 Mortimer Street, London W1T 3JH, UK

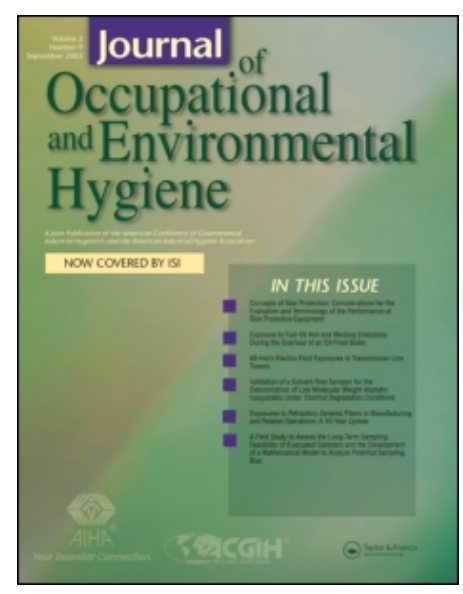

Journal of Occupational and Environmental Hygiene

Publication details, including instructions for authors and subscription information:

http://oeh.informaworld.com/soeh/title content=t713657996

\title{
Rapid Method for Determining Dermal Exposures to Pesticides by Use of Tape Stripping and FTIR Spectroscopy: A Pilot Study
}

Chang-fu Wu ab; Hsiao-Hui Chiu ${ }^{\text {b }}$

a Department of Public Health, National Taiwan University, Taipei, Taiwan ${ }^{\mathrm{b}}$ Institute of Occupational Medicine and Industrial Hygiene/Institute of Environmental Health, National Taiwan University, Taipei, Taiwan

First Published on: 22 October 2007

To cite this Article Wu, Chang-fu and Chiu, Hsiao-Hui(2007)'Rapid Method for Determining Dermal Exposures to Pesticides by Use of Tape Stripping and FTIR Spectroscopy: A Pilot Study',Journal of Occupational and Environmental Hygiene,4:12,952 — 958

To link to this Article: DOI: 10.1080/15459620701719261

URL: http://dx.doi.org/10.1080/15459620701719261

\section{PLEASE SCROLL DOWN FOR ARTICLE}

\footnotetext{
The American Conference of Governmental Industrial Hygienists (http://www.acgih.org/) and the American Industrial Hygiene Association (http://www.aiha.org/) have licensed the Taylor \& Francis Group to publish this article and other materials. To join the American Conference of Governmental Industrial Hygienists visit http://www.acgih.org/Members/. To join the American Industrial Hygiene Association visit http://www.aiha.org/Content/BecomeMember/becomemember-splash.htm.

Full terms and conditions of use: http://oeh.informaworld.com/terms-and-conditions-of-access.pdf

This article maybe used for research, teaching and private study purposes. Any substantial or systematic reproduction, re-distribution, re-selling, loan or sub-licensing, systematic supply or distribution in any form to anyone is expressly forbidden.

The publisher does not give any warranty express or implied or make any representation that the contents will be complete or accurate or up to date. The accuracy of any instructions, formulae and drug doses should be independently verified with primary sources. The publisher shall not be liable for any loss, actions, claims, proceedings, demand or costs or damages whatsoever or howsoever caused arising directly or indirectly in connection with or arising out of the use of this material.
} 


\title{
Rapid Method for Determining Dermal Exposures to Pesticides by Use of Tape Stripping and FTIR Spectroscopy: A Pilot Study
}

\author{
Chang-fu Wu $\mathbf{u}^{1,2}$ and Hsiao-Hui Chiu ${ }^{2}$ \\ ${ }^{1}$ Department of Public Health, National Taiwan University, Taipei, Taiwan \\ ${ }^{2}$ Institute of Occupational Medicine and Industrial Hygiene/Institute of Environmental Health, \\ National Taiwan University, Taipei, Taiwan
}

\begin{abstract}
Tape stripping is a common method for estimating dermal exposure to pesticides because it is relatively noninvasive and easy to use. A major disadvantage is that samples are usually analyzed with gas chromatography, a time-consuming method in terms of sample preparation. In this study, the authors evaluated the feasibility of using Fourier transform infrared spectroscopy (FTIR) to analyze tape stripped samples to provide near real-time dermal exposure estimates. Various exposure scenarios were evaluated, including a single chemical (i.e., chlorpyrifos, a commonly used pesticide) and a mixture of two chemicals (i.e., chlorpyrifos and captan); these were analyzed with or without the human stratum corneum in the samples. Infrared transparent tape was used for sample collection; samples were analyzed using a FTIR spectrometer in the transmittance mode. The partial least squares algorithm was applied to quantify the spectra and the respective $R^{2}$ values for calibration, and test samples were larger than 0.99 and 0.90. The percent divergence of this approach was mostly below $10 \%$, except for several low loading samples. The ANOVA test showed that the stratum corneum's influence on the percent divergence was not significant. Although all the samples evaluated in this study were collected from the same human subject, advantages and feasibility of the strippingFTIR approach were demonstrated.
\end{abstract}

Keywords dermal exposure, FTIR, optical

Address correspondence to: Chang-fu Wu, National Taiwan University, Department of Public Health, Room 717, No.17, Xu-Zhou Rd. Taipei 100, Taiwan, Republic of China; e-mail: changfu@ntu.edu.tw.

\section{INTRODUCTION}

$\mathrm{S}$ kin is the largest organ in the human body and constitutes approximately $7 \%$ of the body's weight. It functions as a barrier to prevent water loss and the entrance of hazardous substances. The stratum corneum (SC) of human skin has an abundance of long-chain, fully saturated acyl groups, which makes the skin an effective hydrophobic barrier. ${ }^{(1)}$ However, some lipophilic chemicals are readily absorbed by the skin. Dermal exposure to hazardous chemicals may cause either local effects (e.g., dermal irritation and sensitization) or systemic effects (e.g., organophosphate poisoning) or may even lead to death.

The following are considered conventional methods for assessing dermal exposure: the surrogate skin technique, the removal technique (e.g., solvent washing or tape stripping), the tracer technique, and biological monitoring. Each method has its advantages as well as its limitations. ${ }^{(2-4)}$ One limitation common to all of these methods (apart from the tracer technique) is that the collected samples need to be sent to a laboratory for further analysis. Although the tracer technique can provide near real-time estimations, it has several limitations, such as that it is not always practical to add a fluorescent tracer to the source materials. Also, some studies have shown that it provides only qualitative results. ${ }^{(5)}$

In recent years, several researchers have started using optical methods, such as attenuated total reflectance Fourier transform infrared (ATR-FTIR) and UV/VIS spectroscopy, to conduct in vivo chemical penetration studies. ${ }^{(6-13)}$ The advantages of these optical methods are that they provide near realtime quantitative results, and the need for sample preparation is minimal. However, one less attractive feature of these optical methods is that they require an extra sampling accessory. Depending on the type of accessory, it could be either relatively expensive (e.g., the ATR crystal) or it would have to be specially designed (e.g., a chalcogens-based glasses IR optical sensor).

In this study, the conventional tape stripping technique is combined with an optical method to overcome some of the above limitations. The tape stripping technique has a good removal efficiency in the range of $70 \%$ to $94 \% .^{(7,14-18)}$ However, this method usually requires samples to be analyzed using gas chromatography, a technique that is time-consuming in terms of sample preparation and the analysis itself.

The authors' approach involved analyzing the tape stripping samples with the transmittance FTIR technique. Compared 
with the ATR-FTIR techniques, this approach has the following advantages: the additional ATR accessory is not required, there is no need to control the contact pressure between the target object and ATR crystal, and it is easier to practice. To evaluate the proposed method, a single pesticide was used without considering the influence of human SC. Then, quantitative analyses of the pesticide samples in the presence of human $\mathrm{SC}$ was performed, and finally, samples containing a mixture of two pesticides were tested.

\section{MATERIALS AND METHODS}

\section{Instrumentation}

All infrared spectra were collected with an FTIR spectrometer (Spectrum 2000, PerkinElmer, Boston, Mass.), which was equipped with a midinfrared deuterated triglycine sulfate detector (MIRTGS) and operated in the transmittance mode. The sampling regime used 50 coaveraged scans at $4 \mathrm{~cm}^{-1}$ resolution. Each single-beam spectrum was collected for $5 \mathrm{~min}$ and was interpolated automatically by the computer software to have the data interval of $1 \mathrm{~cm}^{-1}$. A sampling holder $(4 \mathrm{~cm} \times$ $9 \mathrm{~cm}$ rectangle with a hole of $1.8 \mathrm{~cm}$ in diameter in the center) was placed at the focus of the IR beam. The diameter of the IR beam passing through the center of the holder was $0.8 \mathrm{~cm}$.

\section{Data Acquisition Single Chemical Without SC}

Chlorpyrifos (O,O-diethyl O-3,5,6-trichloro-2-pyridyl phosphorothioate; CAS No. 2921-88-2) was chosen for testing due to its wide use as insecticide in agriculture. Stock solutions were prepared by dissolving pure chlorpyrifos (Sigma-Aldrich, St. Louis, Mo.) in $70 \%$ ethanol. The following procedures were used to obtain a sample spectrum:

- A $3 \mathrm{~cm} \times 3 \mathrm{~cm}$ tape (\#2124C,3M, Minn.) was attached to the sampling holder. Note that the tape has to be IR transparent for the FTIR analysis. After testing several commercially available tapes, it was determined that industrial tape made with polyethylene is remarkably infrared transparent. ${ }^{(19)}$

- A background spectrum was then collected.

- The sampling holder was removed from the instrument, and a certain amount of chemical solution was applied to the tape with a micro pipette.

- After the solvent had evaporated, the sampling holder was replaced, and a sample spectrum was obtained.

Two calibration curves were generated in the high (6.37 to $31.85 \mu \mathrm{g} / \mathrm{cm}^{2}$ ) and the low (0.64 to $3.18 \mu \mathrm{g} / \mathrm{cm}^{2}$ ) exposure loading ranges after reviewing earlier field studies. ${ }^{(20-26)}$ These studies showed that the degree of human exposure to pesticides varies significantly according to the particular pesticide application method. For pure chlorpyrifos, three samples were used for each of the five loading levels to obtain the calibration curves $\left(0.64,1.27,1.91,2.55,3.18 \mu \mathrm{g} / \mathrm{cm}^{2}\right.$ for the low loading range; and $6.37,12.74,19.11,25.48,31.85$ $\mu \mathrm{g} / \mathrm{cm}^{2}$ for the high loading range). For each calibration curve, there were three test samples for each of the four loading levels $\left(0.96,1.59,2.23,2.87 \mu \mathrm{g} / \mathrm{cm}^{2}\right.$ for the low loading range; and $9.55,15.92,22.29,28.66 \mu \mathrm{g} / \mathrm{cm}^{2}$ for the high loading range).

\section{Single Chemical with SC}

The calibration curves of chlorpyrifos with SC in high and low exposure ranges consist of three types of samples $(\mathrm{N}=$ 25 for each calibration curve): (1) Pure chemical samples ( $\mathrm{N}$ $=5$ for each calibration curve): applied chemical to the tape and collected the spectrum (one sample for each of the five loading levels as in the above scenario); (2) SC samples ( $\mathrm{N}=$ 10 for each calibration curve): stripped SC from one subject's forearm with tape and collected the spectrum. Various amounts of SC were obtained by pressing the tape that was fixed to the sampling holder beforehand with different pressure and retention times against the subject's forearm and removing it carefully at a $45^{\circ}$ angle; (3) chemical plus SC samples ( $\mathrm{N}=$ 10 per each calibration curve): chlorpyrifos was applied to the tape used for obtaining the SC samples, then the sample spectra were collected (two samples for each loading level). Twelve test samples (i.e., three samples for each of the four loading levels as in the without-SC scenario above) were also prepared for each calibration curve. The Institutional Review Board of National Taiwan University College of Public Health approved the research protocol.

\section{A Mixture of Two Chemicals}

When applying pesticides in the field, farmers tend to mix two or more chemicals to control a wider range of pests. A mixture of chlorpyrifos and captan $\left(\mathrm{C}_{9} \mathrm{H}_{8} \mathrm{Cl}_{3} \mathrm{NO}_{2} \mathrm{~S}\right.$; CAS No.133-06-2) was evaluated in this study. Stock solutions of captan were prepared by dissolving pure captan (Riedel-de Haën, Seelze, Germany) in $70 \%$ ethanol. The two-chemical scenarios were also tested with or without the SC effects. For the without-SC scenario, calibration samples were prepared by mixing different loading levels of chlorpyrifos $(3.65,6.92$, $\left.9.93 \mu \mathrm{g} / \mathrm{cm}^{2}\right)$ and captan $\left(5.85,11.70,17.56 \mu \mathrm{g} / \mathrm{cm}^{2}\right)$, a total of 15 exposure loading pairs. Test samples $(\mathrm{N}=10)$ were prepared by mixing the two chemicals within the calibration ranges $\left(4.46,8.92\right.$, or $7.80 \mu \mathrm{g} / \mathrm{cm}^{2}$ for chlorpyrifos, and 7.80 , 13.65 , or $15.61 \mu \mathrm{g} / \mathrm{cm}^{2}$ for captan). The calibration samples ( $\mathrm{N}=23$ ) of the with-SC scenario consisted of seven samples of the mixture of the two chemicals, eight SC samples, and eight mixed chemicals with SC samples. Test samples $(\mathrm{N}=10)$ of mixed chlorpyrifos and captan with SC as in the without-SC scenario were prepared for each calibration curve.

\section{Data Analysis}

All spectra data were imported into SAS (V8, SAS Institute Inc., Cary, N.C.) for spectra analysis with the partial least squares (PLS) algorithm. The PLS is a multivariate spectral calibration method that is closely related to Principal Component Regression (PCR). The PLS extracts "factors," or linear combinations of the predictors (i.e., the absorbance data), to explain both the response (i.e., the exposure loading) and 

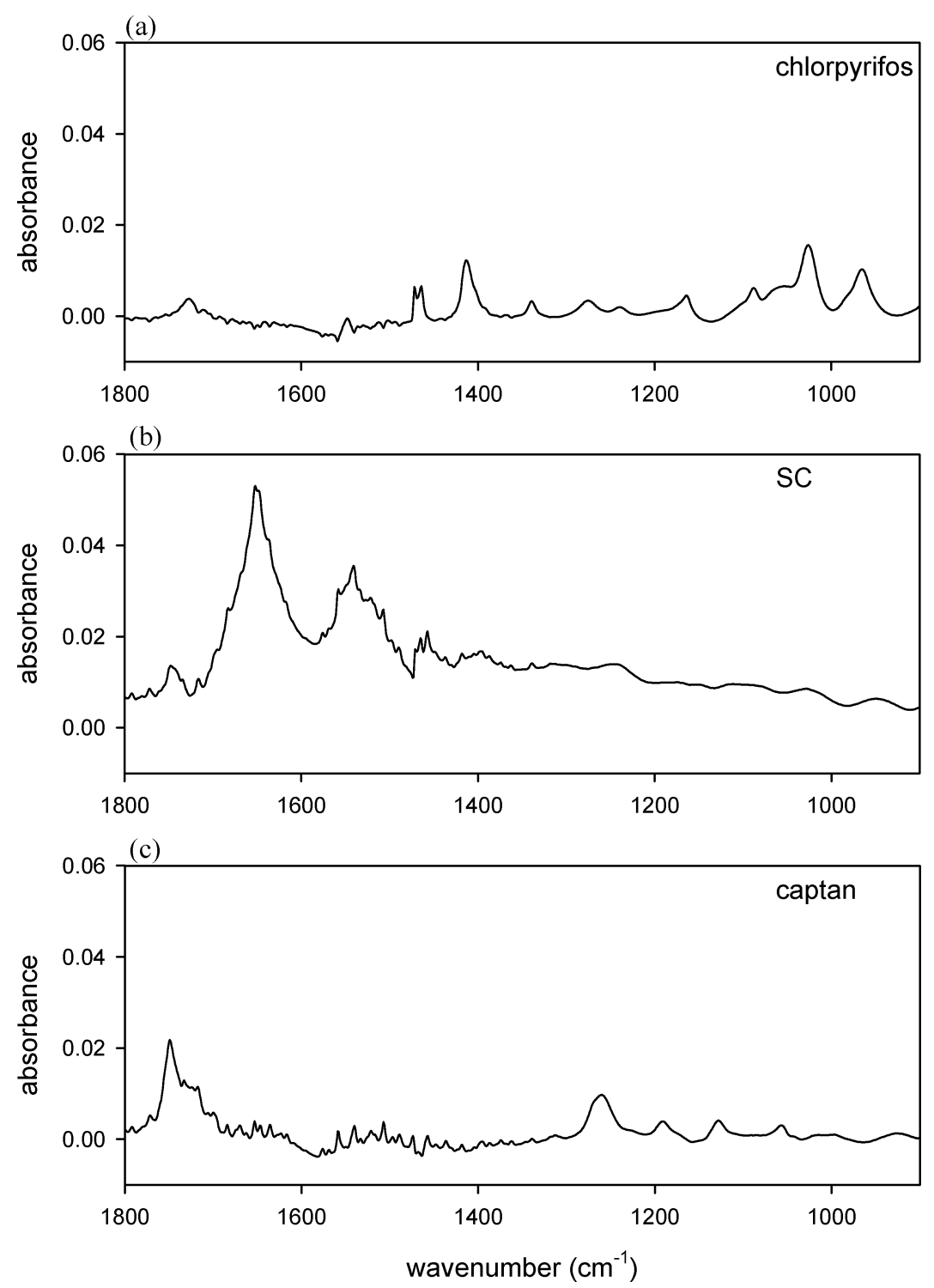

FIGURE 1. The example IR spectra of: (a) $10.03 \mu \mathrm{g} / \mathrm{cm}^{2}$ of chlorpyrifos, (b) 0.05 (unitless) of SC, and (c) $11.70 \mu \mathrm{g} / \mathrm{cm}^{2}$ of captan

predictor variation, while the PCR takes into account only the predictor variation during the spectral decomposing process. This also indicates that the PLS considers errors in both the spectra and the exposure loading estimates. ${ }^{(27)}$

The number of factors included in the final model in this study was determined by one-at-a-time cross-validation based on the predicted residual error sum of squares (PRESS) protocol. The number of factors chosen was the fewest with residuals that are not significantly larger (default p-value of 0.1 ) than the ones of the model with minimum PRESS. The quantification range of $1060 \mathrm{~cm}^{-1}$ to $900 \mathrm{~cm}^{-1}$ was used for chlorpyrifos samples in the single-chemical scenarios, since this range includes the major absorption peaks of the pyridine and the aliphatic phosphate structures and has less influence from the SC (Figure 1). For the two-chemical scenarios, the ranges of $1060 \mathrm{~cm}^{-1}$ to $900 \mathrm{~cm}^{-1}$ and $1300 \mathrm{~cm}^{-1}$ to $1160 \mathrm{~cm}^{-1}$ were used. The two ranges cover the major absorption peaks of chlorpyrifos and captan, respectively (Figure 1).

The quantitative analysis of samples containing both chemicals and SC using the PLS algorithm required that the amount of SC in each calibration sample spectrum be assigned beforehand. The relative amount of SC on the tape was obtained by measuring the absorbance at $1650 \mathrm{~cm}^{-1}$, the largest peak of SC on the IR spectrum (Figure 1). It ranged from 0.024 to 0.167 in the with-SC calibration samples and from 0.012 to 0.139 in the with-SC test samples. The absolute amount of SC is not important as it is not the main interest of this study.

The result of the quantitative analysis was evaluated mainly by calculating the two indicators, \%Divergence and precision, from the test samples. ${ }^{(7,28)}$ The \%Divergence at each test 
loading level was defined as:

$$
\% \text { Divergence }=\frac{\sum_{i=1}^{n}\left|\frac{M_{i}-L}{L}\right| \times 100}{n}
$$

where $M_{i}$ is the measured amount of the $i$ th sample, $L$ is the loaded amount, and $n$ is the number of samples prepared at the $L$ loading level,. The precision of the method was defined as the coefficient of variation $(\mathrm{CV})$ of the quantification results of repeated test samples at the same loading levels.

\section{RESULTS AND DISCUSSION}

\section{Single Chemical}

The limit of detection of chlorpyrifos was $0.07 \mu \mathrm{g} / \mathrm{cm}^{2}$, which is given as 3 times the standard deviation of the noise in the system. It is similar to that $\left(0.066 \mu \mathrm{g} / \mathrm{cm}^{2}\right)$ of the ATR-FTIR method used by Doran et al. ${ }^{(7)}$ and lower than the Environmental Protection Agency (EPA) recommended limit of $1 \mu \mathrm{g} / \mathrm{cm}^{2}$ when using exposure pads for dermal exposure (outdoor) assessment. ${ }^{(29)}$ All the calibration curves of chlorpyrifos in the low and high loading ranges, with or without $\mathrm{SC}$, had a value of $\mathrm{R}^{2}>0.99$ (Table I). The $\mathrm{R}^{2}$ for the test samples were also high $(>0.97)$.

The average \%Divergence for the test samples are shown in Table I. They were $5.1 \%, 5.0 \%, 7.8 \%$, and $9.7 \%$ for the high loading/without-SC, high loading/with-SC, low loading/without-SC, and low loading/with-SC scenarios, respectively. The two highest \%Divergence $(17.7 \%$ and $17.3 \%)$ occurred at the lowest test loading level of $0.96 \mu \mathrm{g} / \mathrm{cm}^{2}$. After excluding these samples, the highest \%Divergence was 9.2\%. On average, the chlorpyrifos test samples in the high loading range had a better \%Divergence than samples in the low loading range. Within the same loading range, these values were approximately the same or just slightly higher after SC was added. This suggests that the errors and variability of the $\mathrm{SC}$ estimates has some influence on the chlorpyrifos analysis, but this influence is not statistically significant (ANOVA p > 0.4 for both loading ranges).

The bias, which has a similar definition as in Eq. 1 but without the absolute sign, ranged from $-5.0 \%$ to $17.7 \%$. After excluding the test samples at the lowest loading level of $0.96 \mu \mathrm{g} / \mathrm{cm}^{2}$, it ranged from $-5.0 \%$ to $9 \%$. No consistent direction, i.e., sign, of bias was observed. As for the precision, it was generally larger for the low loading range than for the high loading range (Table I). However, in the high loading range, the precision was better with the presence of SC because the PLS model was not optimized in terms of the number of factors included in the model. For example, a three-factor (instead of the original two-factor) model for the high loading/without-SC scenario gave an improved \%Divergence $(3.6 \%)$ and precision $(4.3 \%)$.

A sensitivity analysis was conducted by including various numbers of factors (1 to 15 ) in the PLS model (Figure 2). The results showed that extracting more factors did not ensure better performance because the algorithm may over-fit the calibration samples and decrease its prediction power for the test samples. This phenomenon is demonstrated in the low loading scenarios. Through the cross-validation, the 7 factor and 11-factor models were chosen for the low loading without-SC and with-SC samples, respectively. Judging from the minimum \%Divergence values, the 5-factor and 9-factor models were the optimized ones (Figure 2a).

The authors attempted to implement various crossvalidation techniques, such as changing the $\mathrm{p}$-value for the PRESS protocol or holding out two samples at a time, but no universal rule was found that gave the optimized models in all the scenarios. Nevertheless, the \%Divergence and $\mathrm{R}^{2}$ values from the chosen models were still in the vicinity of the ones from the "optimized" models (i.e., the models giving the minimum $\%$ Divergence in Figure 2a). The difference on the $\%$ Divergence was less than $5 \%$. It is also worth pointing out that the PLS models required more factors in the low than in the high loading range. This is partially because the errors and noises in the system have larger influence in the low loading range. For example, in the without-SC scenarios, the first two factors explained $99.6 \%$ and $90.1 \%$ of the response and predictor variation, respectively, in the high loading range, while they explained only $93.5 \%$ and $68.7 \%$ variation in the low loading range.

\section{Two Chemicals}

An example spectrum of pure captan was shown in Figure 1. Its major absorption peak occurred in regions different from that of chlorpyrifos, although some overlapping still existed.

TABLE I. Quantification Results of Chlorpyrifos in the Single Chemical Without-SC and With-SC Scenarios

\begin{tabular}{|c|c|c|c|c|c|c|c|}
\hline \multirow[b]{2}{*}{ Scenario } & \multicolumn{4}{|c|}{ Model Building } & \multicolumn{3}{|c|}{ Model Testing } \\
\hline & $\begin{array}{c}\text { \# Of } \\
\text { Extracted } \\
\text { Factors }\end{array}$ & $\begin{array}{c}\text { Explained \% } \\
\text { of the } \\
\text { Predictor Variation }\end{array}$ & $\begin{array}{c}\text { Explained \% of } \\
\text { the Response } \\
\text { Variation }\end{array}$ & $\mathbf{R}^{2}$ & $\begin{array}{c}\text { \% Divergence } \\
\text { Average } \\
(\text { min, max })\end{array}$ & $\begin{array}{c}\text { Precision } \\
\text { Average } \\
\text { (min, max) }\end{array}$ & $\mathbf{R}^{2}$ \\
\hline High loading without SC & 2 & 90.1 & 99.6 & 0.99 & $5.1(3.2,8.6)$ & $5.2(1.3,9,8)$ & 0.98 \\
\hline High loading with SC & 4 & 99.9 & 92.3 & 0.99 & $5.0(3.7,7.9)$ & $4.2(3.1,5.5)$ & 0.98 \\
\hline Low loading with SC & 11 & 99.9 & 99.9 & 0.99 & $9.7(4.7,17.7)$ & $6.4(5.7,7.8)$ & 0.97 \\
\hline
\end{tabular}



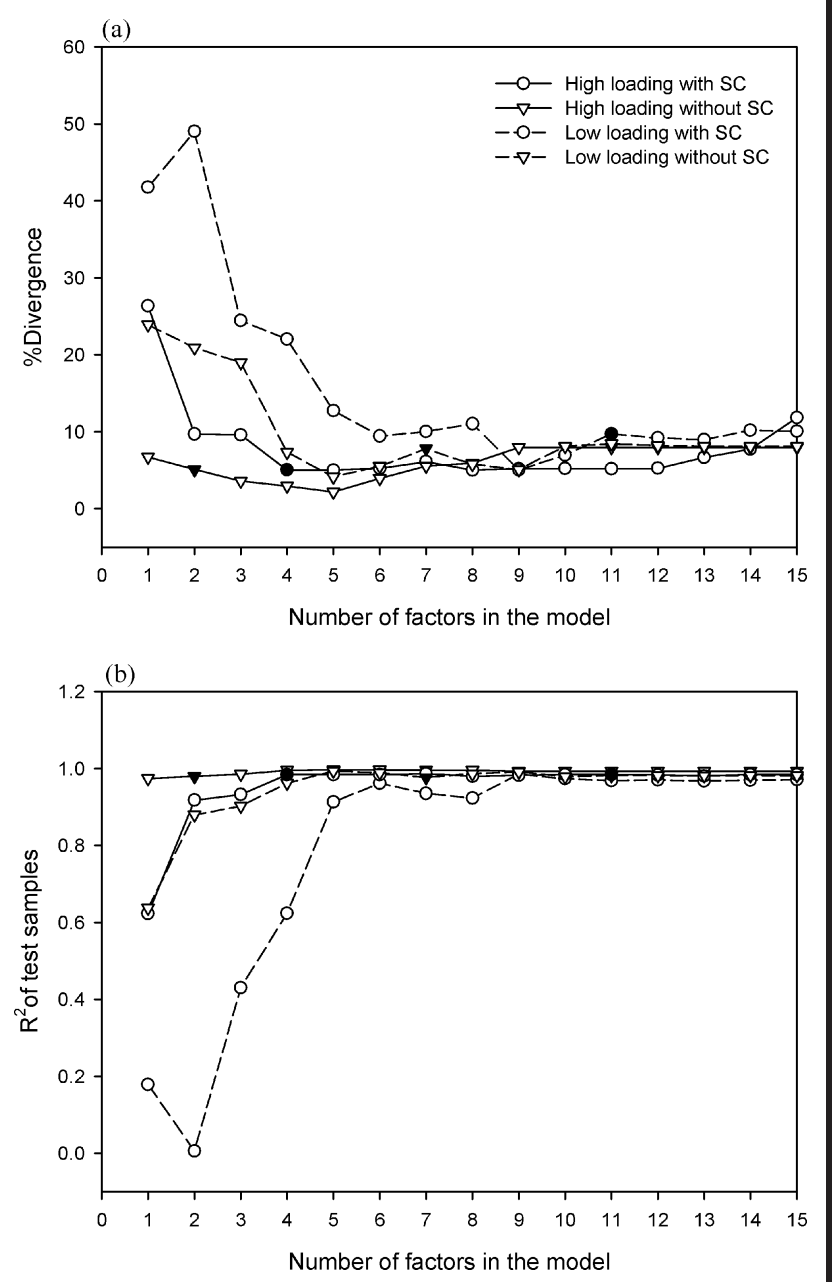

FIGURE 2. Sensitivity analysis results from including different number of extracted factors in the models for the single-chemical scenarios: (a) \%Divergence, (b) $R^{2}$ of the test samples. Filled symbols indicated the statistics from the original models.

The calibration curves for the mixture of chlorpyrifos and captan had $\mathrm{R}^{2}$ values $>0.99$ (Table II). The $\mathrm{R}^{2}$ values for the test samples in the without-SC scenario were higher than 0.96 and were 0.90 and 0.98 for chlorpyrifos and captan, respectively, in the with-SC scenarios (Table II). The average precisions were in the range of $5.8 \%$ to $11 \%$ and the bias ranged from $-10.5 \%$ to $4.4 \%$.

The average \%Divergence was $4.8 \%$ and $5.2 \%$, respectively, for chlorpyrifos and captan in the without-SC scenarios, and $9.6 \%$ and $6.1 \%$ in the with-SC scenarios. The highest $\% \mathrm{Di}-$ vergence was associated with the $4.46 \mu \mathrm{g} / \mathrm{cm}^{2}$ of chlorpyrifos samples. Similar to what was observed for the single chemical scenario, the presence of SC in the samples increased the $\%$ Divergence but not significantly (ANOVA p $>0.1$ ).

Figure 3 shows the sensitivity analysis results of chlorpyrifos from building PLS models with different number of factors. For the without-SC scenario, although the models determined by the cross-validation did not give the lowest $\%$ Divergence, the difference between the ones from the current and the optimized models was only $2.5 \%$. Similar results were observed for captan (data now shown) and the \%Divergence difference between the two models was $2.6 \%$. For the with-SC scenarios, the cross-validation approach successfully chose the optimal number of factors for both the chlorpyrifos (Figure 3) and captan.

The absorbance features of the pesticides tested in this study did not overlap seriously with the stratum corneum's features. When the tape stripping involves dirty skin at work environments or when the spectral overlap of the monitored pesticides and SC is more apparent, this stripping-FTIR approach may have a different degree of performance. The latter condition was demonstrated indirectly by calibrating and quantifying the samples in the mixed-chemical/with-SC scenario with a wider spectral range of $1800 \mathrm{~cm}^{-1}$ to $900 \mathrm{~cm}^{-1}$. This range includes not only the major absorbance features of the chlorpyrifos and captan but also the stratum corenum's features and additional noises. The $\mathrm{R}^{2}$ of the test samples for chlorpyrifos and captan decreased from 0.90 to 0.87 and 0.98 to 0.94 , respectively.

Doran et al. ${ }^{(7)}$ conducted a dermal exposure study by loading the chemicals (i.e., Azinphos-methyl, captan, and chlorpyrifos) onto the ATR-FTIR crystal and then placing the subject's forearm on the crystal before collecting the spectra. The $\%$ Divergence was subsequently estimated to be $4 \%$ for two different samples with exposure loadings of 3 and $5 \mu \mathrm{g} / \mathrm{cm}^{2}$. Except for certain conditions (e.g., samples at low loading

TABLE II. Quantification Results of Mixed Chlorpyrifos and Captan in the Without-SC and With-SC Scenarios

\begin{tabular}{|c|c|c|c|c|c|c|c|c|}
\hline \multirow[b]{2}{*}{ Scenario } & & \multicolumn{4}{|c|}{ Model Building } & \multicolumn{3}{|c|}{ Model Testing } \\
\hline & & $\begin{array}{c}\text { \# Of } \\
\text { Extracted } \\
\text { Factors }\end{array}$ & $\begin{array}{c}\text { Explained \% } \\
\text { of the } \\
\text { Predictor Variation }\end{array}$ & $\begin{array}{l}\text { Explained \% of } \\
\text { the Response } \\
\text { Variation }\end{array}$ & $\mathbf{R}^{2}$ & $\begin{array}{c}\text { \% Divergence } \\
\text { Average } \\
\text { (min, max) }\end{array}$ & $\begin{array}{c}\text { Precision } \\
\text { Average } \\
\text { (min, max) }\end{array}$ & $\mathbf{R}^{2}$ \\
\hline \multirow[t]{2}{*}{ Mixtures without SC } & Chlorpyrifos & 5 & 99.4 & 99.6 & 0.99 & $4.8(1.3,6.1)$ & $5.8(1.8,8.4)$ & 0.97 \\
\hline & Captan & & & & 0.99 & $5.2(5.2,5.2)$ & $6.3(5.0,7.0)$ & 0.96 \\
\hline \multirow[t]{2}{*}{ Mixtures with SC } & Chlorpyrifos & 8 & 99.9 & 99.8 & 0.99 & $9.6(1.2,16.3)$ & $11.1(1.6,18.8)$ & 0.90 \\
\hline & Captan & & & & 0.99 & $6.1(3.0,10.5)$ & $5.8(3.3,8.7)$ & 0.98 \\
\hline
\end{tabular}



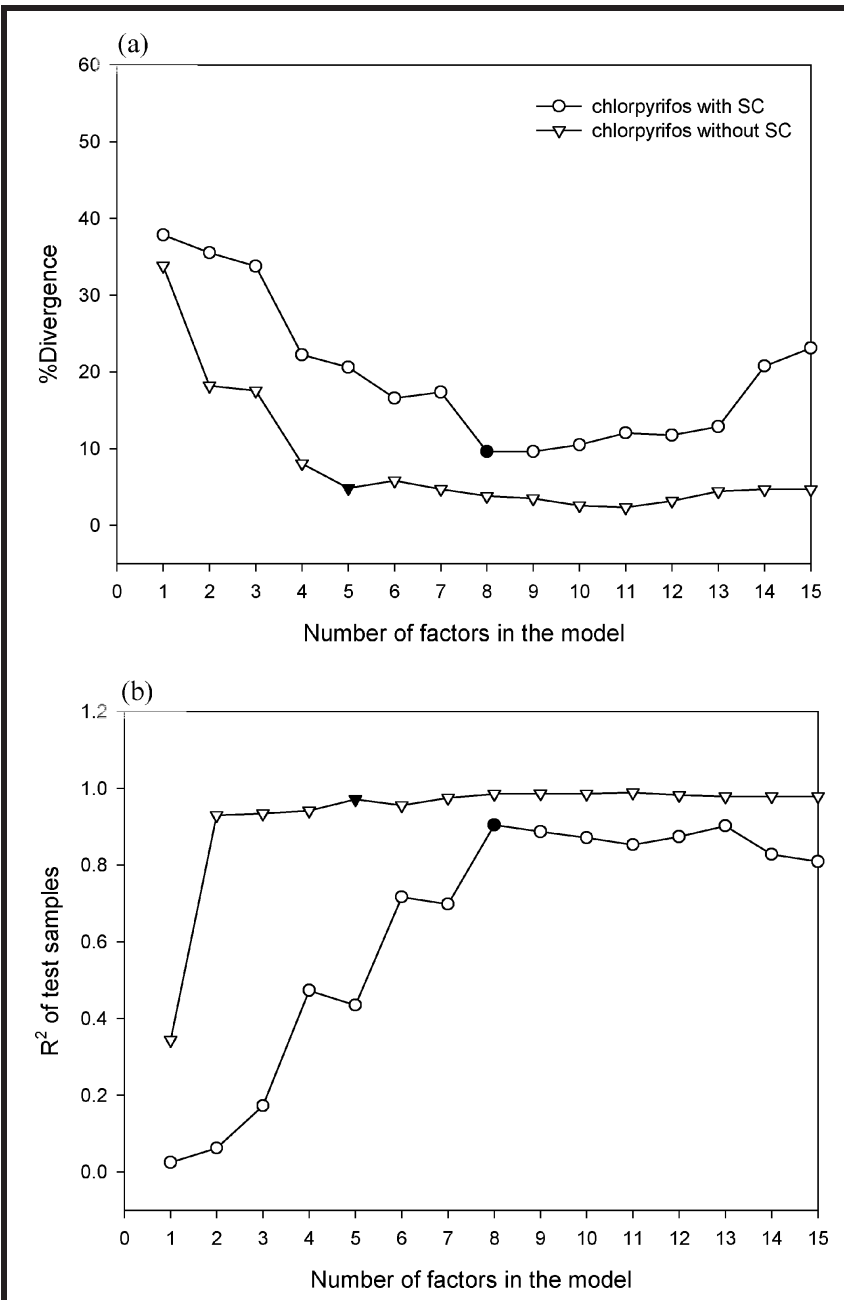

FIGURE 3. Sensitivity analysis results from including different number of extracted factors in the models for chlorpyrifos in the mixed-chemical scenarios: (a) \%Divergence, (b) $R^{2}$ of the test samples. Filled symbols indicated the statistics from the original models.

levels), \%Divergence less than $10 \%$ for most scenarios was obtained. The current stripping-FTIR approach used did not perform as well as the ATR-FTIR method. However, this method is still worth further investigation, especially in field studies, since it has the advantage that tape can be used on most parts of the body and that no ATR sampling accessories are required. Given the good $\mathrm{R}^{2}$ of the test samples but relatively large \%Divergence at the low loading levels, one can consider deploying this system as a screen tool to identify those subjects with high exposures.

As demonstrated in the sensitivity analysis, the crossvalidation approach did not always give the optimized models in this study. Increasing the calibration sample size could help in resolving this issue. In addition, the use of more advanced algorithms with which to quantify the IR spectrum should be considered to achieve better results with the stripping-FTIR approach.
For example, Carden et al. ${ }^{(8)}$ used a novel multivariate data analysis technique (i.e., multivariate curve resolution or MCR) that they applied to the ATR-FTIR spectra of dermal samples. This technique also eliminates the need to assign the amount of SC in the calibration samples when using the PLS algorithm. Instead, skin factors are identified from the MCR's output profiles. One practical drawback of the MCR method is that most standard spectral analysis software packages do not yet contain this method as a standard feature, so that only advanced researchers can run the MCR algorithm.

Because only one human subject was recruited for the present study, the effects of SC variability among different individuals were not evaluated. In theory these effects can be minimized by using each subject's own SC samples as the background spectrum. It should also be possible to compile a database consisting of spectra from multiple subjects to cover most SC variability. Alternatively, an advanced multivariate analysis algorithm could be used to retrieve the SC factor from the collected spectra. Identifying the approach that would provide the most accurate and stable results would require further investigation.

\section{CONCLUSIONS}

This study demonstrated the feasibility of using FTIR spectroscopy for the quantitative analysis of samples collected by tape stripping. This approach combines the advantages of two techniques: (1) the ease and low cost of sample collection of the tape stripping method, and (2) the minimal need for sample preparation and near real-time exposure information obtained by using FTIR spectroscopy. Reasonably good quantitative results were obtained for a single chemical as well as for a mixture of two chemicals with and without the presence of SC.

Although the \%Divergence for certain exposure scenarios was higher than the value previously obtained by Doran et al., ${ }^{(7)}$ the overall \%Divergence was less than $10 \%$. Future studies should consider evaluating the performance of this strippingFTIR approach for multiple subjects under both laboratory and field conditions and verifying the results with conventional stripping-GC analysis approach.

\section{REFERENCES}

1. Elias, M.D.: Epidermal lipids, barrier function, and desquamation. J. Invest. Dermatol. 80:044s-049s (1983).

2. Van Hemmen, J.J., and D.H. Brouwer: Assessment of dermal exposure to chemicals. Sci. Total Environ. 168:131-141 (1995).

3. Schneider, T., J.W. Cherrie, R. Vermeulen, and H. Kromhout: Dermal exposure assessment. Ann. Occup. Hyg. 44:493-499 (2000).

4. Semple, S.: Dermal exposure to chemicals in the workplace: Just how important is skin absorption? J. Occup. Environ. Med. 61:376-382 (2004).

5. Cherrie, J.W., D.H. Brouwer, M. Roff, R. Vermeulen, and $H$. Kromhout: Use of qualitative and quantitative fluorescence techniques to assess dermal exposure. Ann. Occup. Hyg. 44:519-522 (2000). 
6. Stinchcomb, A.L., F. Pirot, G.D. Touraille, A.L. Bunge, and R.H. Guy: Chemical uptake into human stratum corneum in vivo from volatile and non-volatile solvents. Pharma. Res. 16:1288-1293 (1999).

7. Doran, E.M., M.G. Yost, and R.A. Fenske: Measuring dermal exposure to pesticide residues with attenuated total reflectance Fourier transform infrared (ATR-FTIR) spectroscopy. Bull. Environ. Contam. Toxicol. 64:666-672 (2000).

8. Carden, A., M.G. Yost, and R.A. Fenske: Noninvasive method for the assessment of dermal uptake of pesticides using attenuated total reflectance infrared spectroscopy. Appl. Spectrosc. 59:293-299 (2005).

9. Pirot, F., Y.N. Kalia, A.L. Stinchcomb, G. Keating, A. Bunge, and R.H. Guy: Characterization of the permeability barrier of human skin in vivo. In Proc. Nat. Acad. Sci. U.S.A. 94:1562-1567 (1997).

10. Jacobi, U., H.-J. Weigmann, W.S.J. Ulrich, and J. Lademann: Estimation of the relative stratum corneum amount removed by tape stripping. Skin Res. Technol. 11:91-96 (2005).

11. Keirsse, J., C. Boussard-Ple'Del, O. Lore'Al, et al.: IR optical fiber sensor for biomedical applications. Vib. Spectrosc. 32:23-32 (2003).

12. Lafrance, D., L.C. Lands, and D.H. Burns: In vivo lactate measurement in human tissue by near-infrared diffuse reflectance spectroscopy. Vib. Spectrosc. 36:195-202 (2004).

13. Notingher, I., and R.E. Imhof: Mid-infrared in vivo depth-profiling of topical chemicals on skin. Skin Res. Technol. 10:113-121 (2004).

14. Nylander-French, L.A.A.: A tape-stripping method for measuring dermal exposure to multifunctional acrylates.Ann. Occup. Hyg. 44:645651 (2000).

15. Jacobi, U., H.J. Weigmann, J. Ulrich, W. Sterry, and J. Lademann: Estimation of the relative stratum corneum amount removed by tape stripping. Skin Res. Technol. 11:91-96 (2005).

16. Surakka, J., S. Johnsson, G. Rosén, T. Lindh, and T. Fischer: A method for measuring dermal exposure to multifunctional acrylates. J. Environ. Monit. 1:533-540 (1999).

17. Mattorano, D.A., L.L. Kupper, and L.A. Nylander-French: Estimating dermal exposure to jet fuel (naphthalene) using adhesive tape strip samples. Ann. Occup. Hyg. 48:139-146 (2004).
18. Hostýnek, J.J., F. Dreher, T. Nakada, D. Schwindt, A. Anigbogu, and H.I. Maibach: Human stratum corneum adsorption of nickel salts. Acta Derm. Venereol. Suppl. 212:11-18 (2001).

19. Tsilingiris, P.T.: Comparative evaluation of the infrared transmission of polymer films. Energy Conversion Manag. 44:2839-2856 (2003).

20. Warren, N., H.A. Goede, S.C.H.A. Tijssen, R. Oppl, H.J. Schipper, and J.J.V. Hemmen: Deriving default dermal exposure values for use in a risk assessment toolkit for small and medium-sized enterprises. Ann. Occup. Hyg. 47:619-627 (2003).

21. Cattani, M., K. Cena, J. Edwards, and D. Pisaniello: Potential dermal and inhalation exposure to chlorpyrifos in Australian pesticide workers. Ann. Occup. Hyg. 45:299-308 (2001).

22. Brouwer, D.H., M.F. Boeniger, and J. Van Hemmen: Hand wash and manual skin wipes. Ann. Occup. Hyg. 44:501-510 (2000).

23. Geno, P.W., D.E. Camann, H.J. Harding, K. Villalobos, and R.G. Lewis: Handwipe sampling and analysis procedure for the measurement of dermal contact with pesticides. Arch. Environ. Contam. Toxicol. 30:132-138 (1996).

24. Karr, C., P. Demers, L.G. Costa, et al.: Organophosphate pesticide exposure in a group of Washington state orchard applicators. Environ. Res. 59:229-237 (1992).

25. Fenske, R.A., S.G. Birnbaum, M.M. Methner, and R. Soto: Methods for assessing fieldworker hand exposure to pesticides during peach harvesting. Environ. Contam. Toxicol. 43:805-813 (1989).

26. Fenske, R.A., and C. Lu: Determination of handwash removal efficiency: Incomplete removal of the pesticide chlorpyrifos from skin by standard handwash techniques. Am. Ind. Hyg. Assoc. J. 55:425-432 (1994).

27. Brereton, R.G.: Chemometrics: Data Analysis for the Laboratory and Chemical Plant. Chichester, U.K.: John Wiley \& Sons Ltd., 2003.

28. National Institute for Occupational Safety and Health (NIOEH): Guidelines for Air Sampling and Analytical Method Development and Evaluation by E.R. Kennedy, T.J. Fischbach, R. Song, P.M. Eller, and S.A. Shulman (DHHS/NIOSH Pub. No. 95-117), 1995.

29. Environmental Protection Agency (EPA): Occupational and Residential Exposure Test Guidelines (OPPTS 875.1100 Dermal Exposure Outdoor), 1996. 